Proceedings of the Edinburgh Mathematical Society (2002) 45, 333-347 (C)

DOI:10.1017/S0013091501000396 Printed in the United Kingdom

\title{
ASYMPTOTIC STABILITY OF A NEUTRAL DIFFERENTIAL EQUATION
}

\author{
X. H. TANG ${ }^{1}$ AND XINGFU ZOU ${ }^{2}$ \\ ${ }^{1}$ Department of Applied Mathematics, Central South University, Changsha, \\ Hunan 410083, People's Republic of China (xhtang@public.cs.hn.cn) \\ ${ }^{2}$ Department of Mathematics and Statistics, Memorial University of Newfoundland, \\ St John's, NF, Canada A1C 5S7 (xzou@math.mun.ca)
}

(Received 9 March 2001)

\begin{abstract}
The uniform stability of the zero solution and the asymptotic behaviour of all solutions of the neutral delay differential equation

$$
[x(t)-P(t) x(t-\tau)]^{\prime}+Q(t) x(t-\sigma)=0, \quad t \geqslant t_{0},
$$

are investigated, where $\tau, \sigma \in(0, \infty), P \in C\left(\left[t_{0}, \infty\right), \mathbb{R}\right)$, and $Q \in C\left(\left[t_{0}, \infty\right),[0, \infty)\right)$. The obtained sufficient conditions improve the existing results in the literature.
\end{abstract}

Keywords: uniform stability; asymptotic behaviour; neutral delay differential equation

AMS 2000 Mathematics subject classification: Primary 34K20; 34K15; 34K40

\section{Introduction}

Consider the neutral delay differential equation

$$
[x(t)-P(t) x(t-\tau)]^{\prime}+Q(t) x(t-\sigma)=0, \quad t \geqslant t_{0},
$$

where $\tau, \sigma \in(0, \infty), P \in C\left(\left[t_{0}, \infty\right), \mathbb{R}\right)$ and $Q \in C\left(\left[t_{0}, \infty\right),[0, \infty)\right)$.

When $P(t) \equiv 0$, equation (1.1) reduces to

$$
x^{\prime}(t)+Q(t) x(t-\sigma)=0, \quad t \geqslant t_{0},
$$

whose stability of the zero solution has been extensively investigated in the literature (see, for example, $[\mathbf{1}, \mathbf{2}, \mathbf{4}-\mathbf{1 5}]$ ). The best result known to us is the following so-called $\frac{3}{2}$-asymptotic behaviour condition $[\mathbf{1 0}]$ that if

$$
\int_{t_{0}}^{\infty} Q(s) \mathrm{d} s=\infty
$$

and

$$
\limsup _{t \rightarrow \infty} \int_{t-\sigma}^{t} Q(s) \mathrm{d} s<\frac{3}{2}
$$

then every solution of equation (1.2) tends to zero as $t \rightarrow \infty$. 
It was pointed out in $[\mathbf{1 2}]$ that the upper bound $\frac{3}{2}$ in (1.4) is the best possible one for equation (1.2). As an application of the above result, it is easy to conclude that if

$$
\limsup _{t \rightarrow \infty} \int_{t-\sigma}^{t} Q(s) \mathrm{d} s<\frac{3}{2}(1-p)
$$

then every solution of equation (1.1) with $P(t) \equiv p \in(0,1)$ and $\tau=0$ tends to zero as $t \rightarrow \infty$.

When $P(t) \not \equiv 0$, it was shown in [8] that if (1.3) holds and

$$
|P(t)| \equiv p \quad \text { and } \quad 2 p+\limsup _{t \rightarrow \infty} \int_{t-\sigma}^{t} Q(s) \mathrm{d} s<1,
$$

then every solution of equation (1.1) tends to zero as $t \rightarrow \infty$.

For the special case (1.2), (1.6) reduces to

$$
\limsup _{t \rightarrow \infty} \int_{t-\sigma}^{t} Q(s) \mathrm{d} s<1,
$$

which is stronger than (1.4). Therefore, the upper bound 1 in (1.6) is not the best possible one for $(1.1)$. In $[\mathbf{1 1}, \mathbf{1 5}]$, by making use of the double iterative method, the authors developed a condition similar to (1.4) for equation (1.1), which reads

$$
|P(t)| \leqslant p \quad \text { and } \quad 2 p(2-p)+\limsup _{t \rightarrow \infty} \int_{t-\sigma}^{t} Q(s) \mathrm{d} s<\frac{3}{2} .
$$

Since the second inequality in (1.8) is equivalent to

$$
2 p+\limsup _{t \rightarrow \infty} \int_{t-\sigma}^{t} Q(s) \mathrm{d} s<\frac{3}{2}-2 p(1-p),
$$

and $\frac{3}{2}-2 p(1-p)>\frac{3}{2}-\frac{1}{2}=1$, we see that (1.8) improves (1.6). However, condition (1.5) suggests that (1.8) is far from 'the best possible' and there is room for improvement. Therefore, it is desirable to establish some better results, and this constitutes the purpose of this paper. In this paper, we first improve (1.8) to

$$
p<\frac{1}{4} \quad \text { and } \quad 2 p+\limsup _{t \rightarrow \infty} \int_{t-\sigma}^{t} Q(s) \mathrm{d} s<\frac{3}{2},
$$

or

$$
\frac{1}{4} \leqslant p<\frac{1}{2} \quad \text { and } \quad \limsup _{t \rightarrow \infty} \int_{t-\sigma}^{t} Q(s) \mathrm{d} s<\sqrt{2(1-2 p)} .
$$

The fact that (1.9) improves (1.8) is obvious. To see that (1.10) is an improvement of (1.8), we need to show that $\frac{3}{2}-4 p+2 p^{2}<\sqrt{2(1-2 p)}$ for $\frac{1}{4} \leqslant p<\frac{1}{2}$. But $\frac{1}{4} \leqslant p$ implies $2(1-2 p) \leqslant 1$ and, hence, $\sqrt{2(1-2 p)} \geqslant 2(1-2 p)$. Therefore, we only need to show $\frac{3}{2}-4 p+2 p^{2}<2(1-2 p)$, which is just equivalent to $p<\frac{1}{2}$. 
Note that $p<\frac{1}{2}$ is required in both (1.9) and (1.10). We then establish two more new conditions in which $p \geqslant \frac{1}{2}$ is also allowed:

$$
2 p\left(1-\frac{1}{4} p\right)+\limsup _{t \rightarrow \infty} \int_{t-(3 \sigma+(N-1) \tau)}^{t} Q(s) \mathrm{d} s<\frac{3}{2},
$$

where $N$ is a positive integer such that $p+3 p^{N} / 2 \leqslant 1$, and

$$
\limsup _{t \rightarrow \infty} \int_{t-(\sigma+(N-1) \tau)}^{t} Q(s) \mathrm{d} s<\frac{3-4 p^{N}}{2\left(1-p^{N}\right)}(1-p),
$$

where $N$ is a positive integer such that $4 p^{N} \leqslant 1$. The basic ideas of the proofs of (1.11) and (1.12) are decreasing the first term $2 p(2-p)$ by means of increasing the integral interval of $\int_{t-\sigma}^{t} Q(s) \mathrm{d} s$ in (1.8).

It is worth noting that when $\tau=0,(1.12)$ reduces to (1.5) by letting $N \rightarrow \infty$.

\section{2. $P(t)$ is not constant}

Theorem 2.1. Assume that $|P(t)| \leqslant p$ and that

$$
p<\frac{1}{4} \quad \text { and } \quad 2 p+\int_{t}^{t+\sigma} Q(s) \mathrm{d} s \leqslant \frac{3}{2}, \quad t \geqslant t_{0}
$$

or

$$
\frac{1}{4} \leqslant p<\frac{1}{2} \quad \text { and } \quad \int_{t}^{t+\sigma} Q(s) \mathrm{d} s \leqslant \sqrt{2(1-2 p)}, \quad t \geqslant t_{0} .
$$

Then the zero solution of equation (1.1) is uniformly stable.

Proof. If $p=0$, Theorem 2.1 has been proved in [12]. Below, we assume that $0<p<\frac{1}{2}$. Let $\rho=\max \{\tau, \sigma\}, \delta=\min \{\tau, \sigma\}$. Now choose a positive integer $m$ such that $m \delta \geqslant 3 \sigma$. For any $\epsilon>0$, define $\eta=(1-p) \epsilon /(1+p)(2 p+3)^{m}$. We will prove that for any $\bar{t} \geqslant t_{0}, \phi \in C([\bar{t}-\rho, \bar{t}],(-\eta, \eta))$ implies

$$
|x(t ; \bar{t}, \phi)|<\epsilon, \quad t \geqslant \bar{t},
$$

where $x(t ; \bar{t}, \phi)$ denotes the solution of equation (1.1) satisfying the initial condition $x(s ; \bar{t}, \phi)=\phi(s)$ for $s \in[\bar{t}-\rho, \bar{t}]$. For convenience, we denote $x(t)=x(t ; \bar{t}, \phi)$ below and always set

$$
z(t)=x(t)-P(t) x(t-\tau) .
$$

Similar to the proof of $[\mathbf{1 5}$, Theorem 1], we can show that

$$
|x(t)|<(2 p+3)^{m} \eta, \quad t \in[\bar{t}, \bar{t}+m \delta] .
$$

Next we prove (2.3). By way of contradiction, we assume that (2.3) is not true, then by (2.5) there must be some $T>\bar{t}+m \delta$ such that $|x(T)|=\epsilon$ and $|x(t)|<\epsilon$ for $\bar{t} \leqslant t<T$. Without loss of generality, we may suppose that $x(T)=\epsilon$. Thus, we have

$$
z(T)=x(T)-P(T) x(T-\tau)>(1-p) \epsilon>0 .
$$


Again since

$$
z(\bar{t}+m \delta)=x(\bar{t}+m \delta)-P(\bar{t}+m \delta) x(\bar{t}+m \delta-\tau)<(1-p) \epsilon \leqslant z(T),
$$

it follows from (2.6) that there exists $T_{0} \in(\bar{t}+m \delta, T]$ such that $z\left(T_{0}\right)=\max \{z(t)$ : $\bar{t}+m \delta \leqslant t \leqslant T\}$ and $z(t)<z\left(T_{0}\right)$ for $\bar{t}+m \delta \leqslant t<T_{0}$. Set

$$
y(t)=z(t)-p \epsilon, \quad t \geqslant \bar{t} .
$$

Then

$$
\begin{aligned}
-x(t-\sigma) & =-z(t-\sigma)-P(t-\sigma) x(t-\sigma-\tau) \\
& \leqslant-z(t-\sigma)+p \epsilon \\
& =-y(t-\sigma), \quad \bar{t}+\sigma \leqslant t \leqslant T_{0} .
\end{aligned}
$$

It follows from (1.1) and (2.7) that

$$
y^{\prime}(t)=z^{\prime}(t)=-Q(t) x(t-\sigma) \leqslant-Q(t) y(t-\sigma), \quad \bar{t}+\sigma \leqslant t \leqslant T_{0} .
$$

Note that $0<p \leqslant \frac{1}{2}$, it is easy to see that $y\left(T_{0}\right) \geqslant z(T)-p \epsilon>(1-2 p) \epsilon \geqslant 0$. Next we prove $y\left(T_{0}-\sigma\right) \leqslant 0$. Otherwise, $y\left(T_{0}-\sigma\right)>0$. Thus, there is a left neighbour of $T_{0}-\sigma$ which is denoted by $\left(T_{0}-\sigma-h, T_{0}-\sigma\right)$ for some $h>0$, such that $y(t)>0$ for $\left(T_{0}-\sigma-h, T_{0}-\sigma\right)$, and so $y(t-\sigma)>0$ for $\left(T_{0}-h, T_{0}\right)$, and therefore by $(2.8)$, we see that $z(t)$ is not increasing on $\left(T_{0}-h, T_{0}\right)$. This contradicts the definition of $T_{0}$ and so $y\left(T_{0}-\sigma\right) \leqslant 0$. Hence, there exists $\xi \in\left[T_{0}-\sigma, T_{0}\right)$ such that $y(\xi)=0$. From $(2.8)$, we have

$$
y^{\prime}(t) \leqslant Q(t) \epsilon, \quad \bar{t}+\sigma \leqslant t \leqslant T_{0} .
$$

Integrating (2.9), we obtain

$$
-y(t-\sigma) \leqslant \epsilon \int_{t-\sigma}^{\xi} Q(s) \mathrm{d} s, \quad \xi \leqslant t \leqslant T_{0}
$$

Substituting this into (2.8), we have

$$
y^{\prime}(t) \leqslant \epsilon Q(t) \int_{t-\sigma}^{\xi} Q(s) \mathrm{d} s, \quad \xi \leqslant t \leqslant T_{0}
$$

The proof will be complete if we conclude that

$$
(y(T) \leqslant) y\left(T_{0}\right) \leqslant(1-2 p) \epsilon,
$$

which is due to the contradiction of the fact that $y\left(T_{0}\right) \geqslant y(T)>(1-2 p) \epsilon$. There are three possible cases as follows. 
Case $1\left(0<p<\frac{1}{4}\right.$ and $\left.\int_{\xi}^{T_{0}} Q(s) \mathrm{d} s \leqslant 1\right)$. In this case, we have, by integrating (2.10) from $\xi$ to $T_{0}$,

$$
\begin{aligned}
y\left(T_{0}\right) & \leqslant \epsilon \int_{\xi}^{T_{0}} Q(t) \int_{t-\sigma}^{\xi} Q(s) \mathrm{d} s \mathrm{~d} t \\
& =\epsilon \int_{\xi}^{T_{0}} Q(t)\left[\int_{t-\sigma}^{t} Q(s) \mathrm{d} s-\int_{\xi}^{t} Q(s) \mathrm{d} s\right] \mathrm{d} t \\
& \leqslant \epsilon \int_{\xi}^{T_{0}} Q(t)\left[\frac{3}{2}-2 p-\int_{\xi}^{t} Q(s) \mathrm{d} s\right] \mathrm{d} t \\
& =\epsilon\left[\left(\frac{3}{2}-2 p\right) \int_{\xi}^{T_{0}} Q(s) \mathrm{d} s-\frac{1}{2}\left(\int_{\xi}^{T_{0}} Q(s) \mathrm{d} s\right)^{2}\right] \\
& \leqslant(1-2 p) \epsilon .
\end{aligned}
$$

Case $2\left(0<p<\frac{1}{4}\right.$ and $\left.\int_{\xi}^{T_{0}} \boldsymbol{Q}(s) \mathrm{d} s>1\right)$. Choose $T_{1} \in\left(\xi, T_{0}\right)$ such that $\int_{T_{1}}^{T_{0}} Q(s) \mathrm{d} s=1$. Then integrating first (2.9) from $\xi$ to $T_{1}$ and then (2.10) from $T_{1}$ to $T_{0}$, we have

$$
\begin{aligned}
y\left(T_{0}\right) & \leqslant \epsilon \int_{\xi}^{T_{1}} Q(t) \mathrm{d} t+\epsilon \int_{T_{1}}^{T_{0}} Q(t) \int_{t-\sigma}^{\xi} Q(s) \mathrm{d} s \mathrm{~d} t \\
& =\epsilon\left[\int_{T_{1}}^{T_{0}} Q(t) \mathrm{d} t \int_{\xi}^{T_{1}} Q(s) \mathrm{d} s+\int_{T_{1}}^{T_{0}} Q(t) \int_{t-\sigma}^{\xi} Q(s) \mathrm{d} s \mathrm{~d} t\right] \\
& =\epsilon \int_{T_{1}}^{T_{0}} Q(t) \int_{t-\sigma}^{T_{1}} Q(s) \mathrm{d} s \mathrm{~d} t \\
& \leqslant \epsilon\left[\left(\frac{3}{2}-2 p\right) \int_{T_{1}}^{T_{0}} Q(s) \mathrm{d} s-\frac{1}{2}\left(\int_{T_{1}}^{T_{0}} Q(s) \mathrm{d} s\right)^{2}\right] \\
& =(1-2 p) \epsilon .
\end{aligned}
$$

Case $3\left(\frac{1}{4} \leqslant p<\frac{1}{2}\right.$ and $\left.\int_{\xi}^{T_{0}} Q(s) \mathrm{d} s \leqslant \sqrt{\mathbf{2 ( 1 - 2 p )}}\right)$. Integrating (2.10) from $\xi$ to $T_{0}$, we obtain

$$
\begin{aligned}
y\left(T_{0}\right) & \leqslant \epsilon \int_{\xi}^{T_{0}} Q(t) \int_{t-\sigma}^{\xi} Q(s) \mathrm{d} s \mathrm{~d} t \\
& =\epsilon \int_{\xi}^{T_{0}} Q(t)\left[\int_{t-\sigma}^{t} Q(s) \mathrm{d} s-\int_{\xi}^{t} Q(s) \mathrm{d} s\right] \mathrm{d} t \\
& \leqslant \epsilon \int_{\xi}^{T_{0}} Q(t)\left[\sqrt{2(1-2 p)}-\int_{\xi}^{t} Q(s) \mathrm{d} s\right] \mathrm{d} t \\
& =\epsilon\left[\sqrt{2(1-2 p)} \int_{\xi}^{T_{0}} Q(s) \mathrm{d} s-\frac{1}{2}\left(\int_{\xi}^{T_{0}} Q(s) \mathrm{d} s\right)^{2}\right] \\
& \leqslant(1-2 p) \epsilon .
\end{aligned}
$$

Cases 1-3 show that (2.11) is true. And therefore the proof is complete. 
Theorem 2.2. Assume that $|P(t)| \leqslant p$ and (1.3) holds. If (1.9) or (1.10) holds, then every solution of equation (1.1) tends to zero as $t \rightarrow \infty$.

Proof. Let $x(t)$ be any solution of equation (1.1). We shall prove that

$$
\lim _{n \rightarrow \infty} x(t)=0
$$

in two cases where $x(t)$ is non-oscillatory or oscillatory. In the former case, by the proof of [15, Theorem 2], we see that (2.12) holds. In the latter case, by Theorem 2.1, $x(t)$ is bounded. Set $\mu=\lim \sup _{t \rightarrow \infty}|x(t)|$. Then $0 \leqslant \mu<\infty$ and

$$
M=\limsup _{t \rightarrow \infty}|z(t)| \geqslant(1-p) \mu .
$$

The proof will be finished when we prove $\mu=0$. Suppose that $\mu>0$. Then for any $\epsilon \in(0,(1-2 p) \mu)$, there exist $A \in\left(1, \frac{3}{2}\right), B \in(0, \sqrt{2(1-2 p)})$ and $T>t_{0}$ such that

$$
|x(t)|<\mu+\epsilon, \quad t \geqslant T-\rho,
$$

and

$$
\int_{t-\sigma}^{t} Q(s) \mathrm{d} s<\left\{\begin{array}{ll}
A-2 p, & \text { if } p<\frac{1}{4}, \\
B, & \text { if } \frac{1}{4} \leqslant p<\frac{1}{2},
\end{array}\right\} \quad t \geqslant T
$$

Set

$$
y(t)=z(t)-p(\mu+\epsilon), \quad t \geqslant T-\sigma .
$$

Then

$$
\begin{aligned}
-x(t-\sigma) & =-z(t-\sigma)-P(t-\sigma) x(t-\sigma-\tau) \\
& \leqslant-z(t-\sigma)+p(\mu+\epsilon) \\
& =-y(t-\sigma), \quad t \geqslant T .
\end{aligned}
$$

It follows from (1.1) and (2.15) that

$$
y^{\prime}(t)=z^{\prime}(t)=-Q(t) x(t-\sigma) \leqslant-Q(t) y(t-\sigma), \quad t \geqslant T .
$$

Note that $z^{\prime}(t)$ is oscillatory, there is an increasing sequence $\left\{T_{n}\right\}$ such that $T_{n}>T+$ $\tau+2 \sigma, T_{n} \rightarrow \infty,\left|z\left(T_{n}\right)\right| \rightarrow M$ as $n \rightarrow \infty,\left|z\left(T_{n}\right)\right|>(1-p)(\mu-\epsilon)$, and $T_{n}$ is the left local maximum of $|z(t)|$. We may assume that $z\left(T_{n}\right)>0$. The case when $z\left(T_{n}\right)<0$ is similar, and the proof will be omitted. Thus

$$
y\left(T_{n}\right) \geqslant z\left(T_{n}\right)-p(\mu+\epsilon)>(1-2 p)(\mu+\epsilon)-\epsilon>0 .
$$

It is also easy to see that $y\left(T_{n}-\sigma\right) \leqslant 0$ in view of the definition of $T_{n}$. Hence, there exists $\xi_{n} \in\left[T_{n}-\sigma, T_{n}\right)$ such that $y\left(\xi_{n}\right)=0$. From (2.16), we have

$$
y^{\prime}(t) \leqslant Q(t)(\mu+\epsilon), \quad t \geqslant T .
$$


Integrating (2.17), we obtain

$$
-y(t-\sigma) \leqslant(\mu+\epsilon) \int_{t-\sigma}^{\xi_{n}} Q(s) \mathrm{d} s, \quad \xi_{n} \leqslant t \leqslant T_{n} .
$$

Substituting this into (2.16), we have

$$
y^{\prime}(t) \leqslant(\mu+\epsilon) Q(t) \int_{t-\sigma}^{\xi_{n}} Q(s) \mathrm{d} s, \quad \xi_{n} \leqslant t \leqslant T_{n} .
$$

Set

$$
\Lambda= \begin{cases}\max \left\{A-2 p-\frac{1}{2}, \frac{1}{2}\right\}, & \text { if } p<\frac{1}{4}, \\ B^{2} / 2, & \text { if } \frac{1}{4} \leqslant p<\frac{1}{2} .\end{cases}
$$

Then $\Lambda<1-2 p$. We will prove

$$
y\left(T_{n}\right) \leqslant \Lambda(\mu+\epsilon) .
$$

To this end, we consider the following three possible cases.

Case $1\left(0<p<\frac{1}{4}\right.$ and $\left.\int_{\xi_{n}}^{\boldsymbol{T}_{n}} Q(s) \mathrm{d} s \leqslant 1\right)$. In this case, we have by integrating (2.18) from $\xi_{n}$ to $T_{n}$

$$
\begin{aligned}
y\left(T_{n}\right) & \leqslant(\mu+\epsilon) \int_{\xi_{n}}^{T_{n}} Q(t) \int_{t-\sigma}^{\xi_{n}} Q(s) \mathrm{d} s \mathrm{~d} t \\
& =(\mu+\epsilon) \int_{\xi_{n}}^{T_{n}} Q(t)\left(\int_{t-\sigma}^{t} Q(s) \mathrm{d} s-\int_{\xi_{n}}^{t} Q(s) \mathrm{d} s\right) \mathrm{d} t \\
& \leqslant(\mu+\epsilon) \int_{\xi_{n}}^{T_{n}} Q(t)\left(A-2 p-\int_{\xi_{n}}^{t} Q(s) \mathrm{d} s\right) \mathrm{d} t \\
& =(\mu+\epsilon)\left[(A-2 p) \int_{\xi_{n}}^{T_{n}} Q(s) \mathrm{d} s-\frac{1}{2}\left(\int_{\xi_{n}}^{T_{n}} Q(s) \mathrm{d} s\right)^{2}\right] \\
& \leqslant(\mu+\epsilon)\left[\max \{A-2 p, 1\}-\frac{1}{2}\right] \\
& =\Lambda(\mu+\epsilon) .
\end{aligned}
$$

Case $2\left(0<\boldsymbol{p}<\frac{1}{4}\right.$ and $\left.\int_{\xi_{n}}^{\boldsymbol{T}_{n}} \boldsymbol{Q}(s) \mathbf{d} s>\mathbf{1}\right)$. Choose $\eta_{n} \in\left(\xi_{n}, T_{n}\right)$ such that $\int_{\eta_{n}}^{T_{n}} Q(s) \mathrm{d} s=1$. Then integrating first (2.17) from $\xi_{n}$ to $\eta_{n}$ and then (2.18) from $\eta_{n}$ to $T_{n}$, we have

$$
\begin{aligned}
y\left(T_{n}\right) & \leqslant(\mu+\epsilon) \int_{\xi_{n}}^{\eta_{n}} Q(t) \mathrm{d} t+(\mu+\epsilon) \int_{\eta_{n}}^{T_{n}} Q(t) \int_{t-\sigma}^{\xi_{n}} Q(s) \mathrm{d} s \mathrm{~d} t \\
& =(\mu+\epsilon)\left[\int_{\eta_{n}}^{T_{n}} Q(t) \mathrm{d} t \int_{\xi_{n}}^{\eta_{n}} Q(s) \mathrm{d} s+\int_{\eta_{n}}^{T_{n}} Q(t) \int_{t-\sigma}^{\xi_{n}} Q(s) \mathrm{d} s \mathrm{~d} t\right] \\
& =(\mu+\epsilon) \int_{\eta_{n}}^{T_{n}} Q(t) \int_{t-\sigma}^{\eta_{n}} Q(s) \mathrm{d} s \mathrm{~d} t
\end{aligned}
$$




$$
\begin{aligned}
& \leqslant(\mu+\epsilon)\left[(A-2 p) \int_{\eta_{n}}^{T_{n}} Q(s) \mathrm{d} s-\frac{1}{2}\left(\int_{\eta_{n}}^{T_{n}} Q(s) \mathrm{d} s\right)^{2}\right] \\
& =(\mu+\epsilon)\left(A-2 p-\frac{1}{2}\right) \\
& \leqslant \Lambda(\mu+\epsilon) .
\end{aligned}
$$

Case $3\left(\frac{1}{4} \leqslant \boldsymbol{p}<\frac{1}{2}\right.$ and $\left.\int_{\boldsymbol{\xi}_{n}}^{\boldsymbol{T}_{n}} \boldsymbol{Q}(s) \mathbf{d} s \leqslant \boldsymbol{B}\right)$. Integrating (2.18) from $\xi_{n}$ to $T_{n}$, we obtain

$$
\begin{aligned}
y\left(T_{n}\right) & \leqslant(\mu+\epsilon) \int_{\xi_{n}}^{T_{n}} Q(t) \int_{t-\sigma}^{\xi_{n}} Q(s) \mathrm{d} s \mathrm{~d} t \\
& =(\mu+\epsilon) \int_{\xi_{n}}^{T_{n}} Q(t)\left[\int_{t-\sigma}^{t} Q(s) \mathrm{d} s-\int_{\xi_{n}}^{t} Q(s) \mathrm{d} s\right] \mathrm{d} t \\
& \leqslant(\mu+\epsilon) \int_{\xi_{n}}^{T_{n}} Q(t)\left(B-\int_{\xi_{n}}^{t} Q(s) \mathrm{d} s\right) \mathrm{d} t \\
& =(\mu+\epsilon)\left[B \int_{\xi_{n}}^{T_{n}} Q(s) \mathrm{d} s-\frac{1}{2}\left(\int_{\xi_{n}}^{T_{n}} Q(s) \mathrm{d} s\right)^{2}\right] \\
& \leqslant \frac{1}{2} B^{2}(\mu+\epsilon) \\
& =\Lambda(\mu+\epsilon) .
\end{aligned}
$$

Cases $1-3$ show that (2.19) is true. From (2.19), we have

$$
z\left(T_{n}\right) \leqslant(\Lambda+p)(\mu+\epsilon) .
$$

Let $n \rightarrow \infty$ and $\epsilon \rightarrow 0$ in the above. We see that

$$
M=\limsup _{n \rightarrow \infty} z\left(T_{n}\right) \leqslant(\Lambda+p) \mu<(1-p) \mu,
$$

which contradicts (2.13). Therefore, $\mu=0$, and so the proof is complete.

If $P(t)$ is not negative, then we have the following two theorems.

Theorem 2.3. Assume that $0 \leqslant P(t) \leqslant p$ and that there exists a positive integer $N$ such that $p+3 p^{N} / 2 \leqslant 1$ and

$$
2 p\left(1-\frac{1}{4} p\right)+\int_{t}^{t+3 \sigma+(N-1) \tau} Q(s) \mathrm{d} s \leqslant \frac{3}{2}, \quad t \geqslant t_{0} .
$$

Then the zero solution of equation (1.1) is uniformly stable.

Proof. If $p=0$, Theorem 2.3 has been proved in [12]. Below, we assume that $0<$ $p<1$. Let $\rho=\max \{\tau, \sigma\}, \delta=\min \{\tau, \sigma\}$. Now choose a positive integer $m$ such that $m \delta \geqslant 2(3 \sigma+N \tau)$. For any $\epsilon>0$, define $\eta=(1-p) \epsilon /(1+p)(2 p+3)^{m}$. We will prove that for any $\bar{t} \geqslant t_{0}, \phi \in C([\bar{t}-\rho, \bar{t}],(-\eta, \eta))$ implies that $x(t)$ satisfies $(2.3)$, where $x(t)=x(t ; \bar{t}, \phi)$ denotes the solution of equation (1.1) satisfying the initial condition $x(s ; \bar{t}, \phi)=\phi(s)$ for 
$s \in[\bar{t}-\rho, \bar{t}]$. First, similar to the proof of Theorem 2.1, we can prove that (2.5) holds. Obviously, (2.5) implies that (2.3) holds for $\bar{t} \leqslant t \leqslant \bar{t}+m \delta$. Next, we prove that (2.3) holds for $t>\bar{t}+m \delta$. By way of contradiction, we assume that there is some $T>\bar{t}+m \delta$ such that $|x(T)|=\epsilon$ and $|x(t)|<\epsilon$ for $\bar{t} \leqslant t<T$. Without loss of generality, we may suppose that $x(T)=\epsilon$. Thus, (2.6) is true and there exists $T_{0} \in(\bar{t}+m \delta, T]$ such that $z\left(T_{0}\right)=\max \{z(t): \bar{t}+m \delta \leqslant t \leqslant T\}$ and $z(t)<z\left(T_{0}\right)$ for $\bar{t}+m \delta \leqslant t<T_{0}$. There are now two possibilities:

$$
z(t)>0, \quad t \in\left[T_{0}-(3 \sigma+(N-1) \tau), T_{0}\right],
$$

or there exists $\xi \in\left[T_{0}-(3 \sigma+(N-1) \tau), T_{0}\right]$ such that

$$
z(\xi)=0, \quad z(t)>0, \quad t \in\left(\xi, T_{0}\right] .
$$

In the former case, we have

$$
-x(t)<p^{i} \epsilon, \quad t \in\left[T_{0}-(3 \sigma+(N-i) \tau), T_{0}\right], \quad i=1,2, \ldots, N,
$$

and

$$
\begin{aligned}
z^{\prime}(t) & =Q(t)[-z(t-\sigma)-P(t-\sigma) x(t-\sigma-\tau)] \\
& \leqslant-Q(t)\left[z(t-\sigma)-p^{N} \epsilon\right], \quad t \in\left[T_{0}-2 \sigma, T_{0}\right] .
\end{aligned}
$$

Set

$$
y(t)=z(t)-p^{N} \epsilon, \quad t \geqslant \bar{t}
$$

Then

$$
y^{\prime}(t)=z^{\prime}(t)=-Q(t) x(t-\sigma) \leqslant-Q(t) y(t-\sigma), \quad t \in\left[T_{0}-2 \sigma, T_{0}\right] .
$$

Similar to the proof of Theorem 2.1, it is easy to conclude from (2.23) that $y\left(T_{0}-\sigma\right) \leqslant$ 0 . Note that $y\left(T_{0}\right) \geqslant z(T)-p^{N} \epsilon>\left(1-p-p^{N}\right) \epsilon>0$. It follows that there exists $\zeta \in\left[T_{0}-\sigma, T_{0}\right)$ such that $y(\zeta)=0$. From (2.22) and (2.23), we have

$$
y^{\prime}(t) \leqslant Q(t) p^{N} \epsilon, \quad t \in\left[T_{0}-2 \sigma, T_{0}\right]
$$

Integrating (2.24) from $\zeta$ to $T_{0}$, we obtain

$$
-y(t-\sigma) \leqslant p^{N} \epsilon \int_{t-\sigma}^{\zeta} Q(s) \mathrm{d} s, \quad t \in\left[T_{0}-\sigma, T_{0}\right] .
$$

Substituting this into (2.23), we have

$$
y^{\prime}(t) \leqslant p^{N} \epsilon Q(t) \int_{t-\sigma}^{\zeta} Q(s) \mathrm{d} s, \quad t \in\left[T_{0}-\sigma, T_{0}\right] .
$$

There are two possible subcases. 
Subcase $1\left(\int_{\zeta}^{T_{0}} Q(s) \mathrm{d} s \leqslant \mathbf{1}-\boldsymbol{p}\right)$. In this case, we have, by integrating (2.25) from $\zeta$ to $T_{0}$,

$$
\begin{aligned}
y\left(T_{0}\right) & \leqslant p^{N} \epsilon \int_{\zeta}^{T_{0}} Q(t) \int_{t-\sigma}^{\zeta} Q(s) \mathrm{d} s \mathrm{~d} t \\
& =p^{N} \epsilon \int_{\zeta}^{T_{0}} Q(t)\left[\int_{t-\sigma}^{t} Q(s) \mathrm{d} s-\int_{\zeta}^{t} Q(s) \mathrm{d} s\right] \mathrm{d} t \\
& \leqslant p^{N} \epsilon \int_{\zeta}^{T_{0}} Q(t)\left[\frac{(1-p)(3-p)}{2}-\int_{\zeta}^{t} Q(s) \mathrm{d} s\right] \mathrm{d} t \\
& =p^{N} \epsilon\left[\frac{(1-p)(3-p)}{2} \int_{\zeta}^{T_{0}} Q(s) \mathrm{d} s-\frac{1}{2}\left(\int_{\zeta}^{T_{0}} Q(s) \mathrm{d} s\right)^{2}\right] \\
& \leqslant(1-p)^{2} p^{N} \epsilon \leqslant\left(1-p-p^{N}\right) \epsilon .
\end{aligned}
$$

Subcase $2\left(\int_{\zeta}^{\boldsymbol{T}_{0}} \boldsymbol{Q}(s) \mathrm{d} s>\mathbf{1}-\boldsymbol{p}\right)$. Choose $T_{1} \in\left(\zeta, T_{0}\right)$ such that $\int_{T_{1}}^{T_{0}} Q(s) \mathrm{d} s=1-p$. Then, integrating first (2.24) from $\zeta$ to $T_{1}$ and then (2.25) from $T_{1}$ to $T_{0}$, we have

$$
\begin{aligned}
y\left(T_{0}\right) & \leqslant p^{N} \epsilon \int_{\zeta}^{T_{1}} Q(t) \mathrm{d} t+p^{N} \epsilon \int_{T_{1}}^{T_{0}} Q(t) \int_{t-\sigma}^{\zeta} Q(s) \mathrm{d} s \mathrm{~d} t \\
& =p^{N} \epsilon\left[\int_{T_{1}}^{T_{0}} Q(t) \mathrm{d} t \int_{\zeta}^{T_{1}} Q(s) \mathrm{d} s+p \int_{\zeta}^{T_{1}} Q(s) \mathrm{d} s+\int_{T_{1}}^{T_{0}} Q(t) \int_{t-\sigma}^{\zeta} Q(s) \mathrm{d} s \mathrm{~d} t\right] \\
& =p^{N} \epsilon\left[\int_{T_{1}}^{T_{0}} Q(t) \int_{t-\sigma}^{T_{1}} Q(s) \mathrm{d} s \mathrm{~d} t+p\left(\int_{\zeta}^{T_{0}} Q(s) \mathrm{d} s-\int_{T_{1}}^{T_{0}} Q(s) \mathrm{d} s\right)\right] \\
& \leqslant p^{N} \epsilon\left[\frac{(1-p)(3-p)}{2} \int_{T_{1}}^{T_{0}} Q(s) \mathrm{d} s-\frac{1}{2}\left(\int_{T_{1}}^{T_{0}} Q(s) \mathrm{d} s\right)^{2}\right. \\
& \left.\quad+p\left(\frac{(1-p)(3-p)}{2}-\int_{T_{1}}^{T_{0}} Q(s) \mathrm{d} s\right)\right] \\
& =(1-p)^{2} p^{N} \epsilon \leqslant\left(1-p-p^{N}\right) \epsilon .
\end{aligned}
$$

Subcases 1 and 2 show that

$$
y\left(T_{0}\right) \leqslant\left(1-p-p^{N}\right) \epsilon,
$$

which contradicts the fact that $y\left(T_{0}\right) \geqslant y(T)>\left(1-p-p^{N}\right) \epsilon$.

In the latter case, we have

$$
z^{\prime}(t) \leqslant Q(t) \epsilon, \quad \bar{t} \leqslant t \leqslant T_{0}
$$

and

$$
z^{\prime}(t) \leqslant \epsilon Q(t)\left[p+\int_{t-(3 \sigma+(N-1) \tau)}^{\xi} Q(s) \mathrm{d} s\right], \quad \xi \leqslant t \leqslant T_{0} .
$$

There are two possible subcases as follows. 
Subcase $1\left(\int_{\xi}^{T_{0}} \boldsymbol{Q}(s) \mathrm{d} s \leqslant \mathbf{1}-\boldsymbol{p}\right)$. In this case, we have by integrating (2.28) from $\xi$ to $T_{0}$

$$
\begin{aligned}
z\left(T_{0}\right) & \leqslant \epsilon \int_{\xi}^{T_{0}} Q(t)\left[p+\int_{t-3 \sigma-(N-1) \tau}^{\xi} Q(s) \mathrm{d} s\right] \mathrm{d} t \\
& =\epsilon \int_{\xi}^{T_{0}} Q(t)\left[p+\int_{t-3 \sigma-(N-1) \tau}^{t} Q(s) \mathrm{d} s-\int_{\xi}^{t} Q(s) \mathrm{d} s\right] \mathrm{d} t \\
& \leqslant \epsilon \int_{\xi}^{T_{0}} Q(t)\left[p+\frac{(1-p)(3-p)}{2}-\int_{\xi}^{t} Q(s) \mathrm{d} s\right] \mathrm{d} t \\
& =\epsilon\left[\frac{3-2 p+p^{2}}{2} \int_{\xi}^{T_{0}} Q(s) \mathrm{d} s-\frac{1}{2}\left(\int_{\xi}^{T_{0}} Q(s) \mathrm{d} s\right)^{2}\right] \\
& \leqslant(1-p) \epsilon .
\end{aligned}
$$

Subcase $2\left(\int_{\boldsymbol{\xi}}^{\boldsymbol{T}_{\mathbf{0}}} \boldsymbol{Q}(\boldsymbol{s}) \mathrm{d} \boldsymbol{s}>\mathbf{1}-\boldsymbol{p}\right)$. Choose $T_{2} \in\left(\xi, T_{0}\right)$ such that $\int_{T_{2}}^{T_{0}} Q(s) \mathrm{d} s=1-p$. Then integrating first (2.27) from $\xi$ to $T_{2}$ and then (2.28) from $T_{2}$ to $T_{0}$, we have

$$
\begin{aligned}
z\left(T_{0}\right) \leqslant & \epsilon \int_{\xi}^{T_{2}} Q(t) \mathrm{d} t+\epsilon \int_{T_{2}}^{T_{0}} Q(t)\left[p+\int_{t-3 \sigma-(N-1) \tau}^{\xi} Q(s) \mathrm{d} s\right] \mathrm{d} t \\
= & \epsilon\left[\int_{T_{2}}^{T_{0}} Q(t) \mathrm{d} t \int_{\xi}^{T_{2}} Q(s) \mathrm{d} s+p \int_{\xi}^{T_{0}} Q(s) \mathrm{d} s\right. \\
& \left.\quad+\int_{T_{2}}^{T_{0}} Q(t) \int_{t-3 \sigma-(N-1) \tau}^{\xi} Q(s) \mathrm{d} s \mathrm{~d} t\right] \\
= & \epsilon\left[\int_{T_{2}}^{T_{0}} Q(t) \int_{t-3 \sigma-(N-1) \tau}^{T_{2}} Q(s) \mathrm{d} s \mathrm{~d} t+p \int_{\xi}^{T_{0}} Q(s) \mathrm{d} s\right] \\
\leqslant & \epsilon\left[\frac{(1-p)(3-p)}{2} \int_{T_{2}}^{T_{0}} Q(s) \mathrm{d} s-\frac{1}{2}\left(\int_{T_{2}}^{T_{0}} Q(s) \mathrm{d} s\right)^{2}+p \frac{(1-p)(3-p)}{2}\right] \\
= & (1-p) \epsilon .
\end{aligned}
$$

Subcases 1 and 2 show that

$$
z\left(T_{0}\right) \leqslant(1-p) \epsilon
$$

which contradicts the fact that $z\left(T_{0}\right) \geqslant z(T)>(1-p) \epsilon$. The proof is complete.

Similar to Theorem 2.2 and Theorem 2.3 and applying Lemma 6.4.1 from [3], we can show the following asymptotic behaviour theorem.

Theorem 2.4. Assume that $0 \leqslant P(t) \leqslant p$ and there exists a positive integer $N$ such that $p+3 p^{N} / 2 \leqslant 1$. If (1.3) and (1.11) hold, then every solution of equation (1.1) tends to zero as $t \rightarrow \infty$. 


\section{3. $P(t)$ is constant}

Theorem 3.1. Assume that $|P(t)| \equiv p$ and that there exists a positive integer $N$ such that $4 p^{N} \leqslant 1$ and

$$
\int_{t}^{t+\sigma+(N-1) \tau} Q(s) \mathrm{d} s \leqslant \frac{3-4 p^{N}}{2\left(1-p^{N}\right)}(1-p), \quad t \geqslant t_{0} .
$$

Then the zero solution of equation (1.1) is uniformly stable.

Proof. If $p=0$, Theorem 3.1 has been proved in [12]. Below, we assume that $0<$ $4 p^{N} \leqslant 1$. Let $\rho=\max \{\tau, \sigma\}, \delta=\min \{\tau, \sigma\}$. Now choose a positive integer $m$ such that $m \delta \geqslant 2(\sigma+N \tau)$. For any $\epsilon>0$, define $\eta=(1-p) \epsilon /(1+p)(2 p+3)^{m}$. We will prove that for any $\bar{t} \geqslant t_{0}, \phi \in C([\bar{t}-\rho, \bar{t}],(-\eta, \eta))$ implies that $x(t)$ satisfies $(2.3)$, where $x(t)=x(t ; \bar{t}, \phi)$ denotes the solution of equation (1.1) satisfying the initial condition $x(s ; \bar{t}, \phi)=\phi(s)$ for $s \in[\bar{t}-\rho, \bar{t}]$. Similar to the proof of Theorem 2.1, we can prove that (2.5) holds. Next, we prove (2.3). By way of contradiction, we assume that (2.3) is not true, then by (2.5) there must be some $T>\bar{t}+m \delta$ such that $|x(T)|=\epsilon$ and $|x(t)|<\epsilon$ for $\bar{t} \leqslant t<T$. Without loss of generality, we may suppose that $x(T)=\epsilon$. Thus, (2.6) is true and there exists $T_{0} \in(\bar{t}+m \delta, T]$ such that $z\left(T_{0}\right)=\max \{z(t): \bar{t}+m \delta \leqslant t \leqslant T\}$ and $z(t)<z\left(T_{0}\right)$ for $\bar{t}+m \delta \leqslant t<T_{0}$. Set

$$
y(t)=z(t)-\frac{1-p}{1-p^{N}} p^{N} \epsilon, \quad t \geqslant \bar{t} .
$$

Then

$$
\begin{aligned}
-x(t-\sigma) & =-\sum_{i=0}^{N-1} p^{i} z(t-\sigma-i \tau)-p^{N} x(t-\sigma-N \tau) \\
& \leqslant-\sum_{i=0}^{N-1} p^{i} z(t-\sigma-i \tau)+p^{N} \epsilon \\
& =-\sum_{i=0}^{N-1} p^{i} y(t-\sigma-i \tau), \quad T_{0}-\sigma-(N-1) \tau \leqslant t \leqslant T_{0} .
\end{aligned}
$$

It follows from (1.1) and (3.2) that

$$
y^{\prime}(t)=z^{\prime}(t)=-Q(t) x(t-\sigma), \quad t \geqslant \bar{t}
$$

and

$$
y^{\prime}(t) \leqslant-Q(t) \sum_{i=0}^{N-1} p^{i} y(t-\sigma-i \tau), \quad T_{0}-\sigma-(N-1) \tau \leqslant t \leqslant T_{0} .
$$

It is easy to see that $y\left(T_{0}\right) \geqslant z(T)-(1-p) p^{N} \epsilon /\left(1-p^{N}\right)>(1-p)\left[1-p^{N} /\left(1-p^{N}\right)\right] \epsilon \geqslant 0$. Next we prove that there exists $j \in\{0,1, \ldots, N-1\}$ such that $y\left(T_{0}-\sigma-j \tau\right) \leqslant 0$. Otherwise, $y\left(T_{0}-\sigma-i \tau\right)>0, i=0,1, \ldots, N-1$. Thus, there is a left neighbour of 
$T_{0}-\sigma-i \tau$ which is denoted by $\left(T_{0}-\sigma-i \tau-h, T_{0}-\sigma-i \tau\right)$ for some $h>0$, such that $y(t)>0$ for $\left(T_{0}-\sigma-i \tau-h, T_{0}-\sigma-i \tau\right)$, and so $y(t-\sigma-i \tau)>0$ for $\left(T_{0}-h, T_{0}\right)$ and $i=0,1, \ldots, N-1$, and therefore by (3.4), we see that $y(t)$ is not increasing on $\left(T_{0}-h, T_{0}\right)$. This contradicts the definition of $T_{0}$ and so $y\left(T_{0}-\sigma-j \tau\right) \leqslant 0$ for some $j \in\{0,1, \ldots, N-1\}$. Hence, there exists $\xi \in\left[T_{0}-\sigma-(N-1) \tau, T_{0}\right)$ such that $y(\xi)=0$ and $y(t)>0, t \in\left(\xi, T_{0}\right]$. From (3.3), we have

$$
y^{\prime}(t) \leqslant Q(t) \epsilon, \quad \bar{t} \leqslant t \leqslant T_{0}
$$

If $t-\sigma-i \tau \leqslant \xi$ for $t \in\left[T_{0}-\sigma-(N-1) \tau, T_{0}\right]$, then integrating (3.5) we obtain

$$
-y(t-\sigma-i \tau) \leqslant \epsilon \int_{t-\sigma-(N-1) \tau}^{\xi} Q(s) \mathrm{d} s, \quad t-\sigma-i \tau \leqslant \xi .
$$

If $\xi<t-\sigma-i \tau \leqslant T_{0}$ for $t \in\left[T_{0}-\sigma-(N-1) \tau, T_{0}\right]$, then

$$
-y(t-\sigma-i \tau)<0 \leqslant \epsilon \int_{t-\sigma-(N-1) \tau}^{\xi} Q(s) \mathrm{d} s, \quad \xi<t-\sigma-i \tau \leqslant T_{0} .
$$

Substituting these into (3.4), we have

$$
y^{\prime}(t) \leqslant \epsilon \frac{1-p^{N}}{1-p} Q(t) \int_{t-\sigma-(N-1) \tau}^{\xi} Q(s) \mathrm{d} s, \quad \xi \leqslant t \leqslant T_{0}
$$

There are two possible cases as follows.

Case $1\left(\left(\left(1-p^{N}\right) /(1-p)\right) \int_{\xi}^{T_{0}} Q(s) \mathrm{d} s \leqslant 1\right)$. In this case, we have by integrating (3.6) from $\xi$ to $T_{0}$

$$
\begin{aligned}
y\left(T_{0}\right) & \leqslant \frac{1-p^{N}}{1-p} \epsilon \int_{\xi}^{T_{0}} Q(t) \int_{t-\sigma-(N-1) \tau}^{\xi} Q(s) \mathrm{d} s \mathrm{~d} t \\
& =\frac{1-p^{N}}{1-p} \epsilon \int_{\xi}^{T_{0}} Q(t)\left[\int_{t-\sigma-(N-1) \tau}^{t} Q(s) \mathrm{d} s-\int_{\xi}^{t} Q(s) \mathrm{d} s\right] \mathrm{d} t \\
& \leqslant \frac{1-p^{N}}{1-p} \epsilon \int_{\xi}^{T_{0}} Q(t)\left[\frac{3-4 p^{N}}{2\left(1-p^{N}\right)}(1-p)-\int_{\xi}^{t} Q(s) \mathrm{d} s\right] \mathrm{d} t \\
& =\frac{1-p^{N}}{1-p} \epsilon\left[\frac{3-4 p^{N}}{2\left(1-p^{N}\right)}(1-p) \int_{\xi}^{T_{0}} Q(s) \mathrm{d} s-\frac{1}{2}\left(\int_{\xi}^{T_{0}} Q(s) \mathrm{d} s\right)^{2}\right] \\
& \leqslant \frac{1-2 p^{N}}{1-p^{N}}(1-p) \epsilon .
\end{aligned}
$$

Case $2\left(\left(\left(1-p^{N}\right) /(1-p)\right) \int_{\xi}^{\boldsymbol{T}_{\mathbf{0}}} \boldsymbol{Q}(s) \mathbf{d} s>\mathbf{1}\right)$. Choose $T_{1} \in\left(\xi, T_{0}\right)$ such that

$$
\frac{1-p^{N}}{1-p} \int_{T_{1}}^{T_{0}} Q(s) \mathrm{d} s=1
$$


Then integrating first (3.5) from $\xi$ to $T_{1}$ and then (3.6) from $T_{1}$ to $T_{0}$, we have

$$
\begin{aligned}
y\left(T_{0}\right) & \leqslant \epsilon \int_{\xi}^{T_{1}} Q(t) \mathrm{d} t+\frac{1-p^{N}}{1-p} \epsilon \int_{T_{1}}^{T_{0}} Q(t) \int_{t-\sigma-(N-1) \tau}^{\xi} Q(s) \mathrm{d} s \mathrm{~d} t \\
& =\frac{1-p^{N}}{1-p} \epsilon \int_{T_{1}}^{T_{0}} Q(t) \mathrm{d} t \int_{\xi}^{T_{1}} Q(s) \mathrm{d} s+\frac{1-p^{N}}{1-p} \epsilon \int_{T_{1}}^{T_{0}} Q(t) \int_{t-\sigma-(N-1) \tau}^{\xi} Q(s) \mathrm{d} s \mathrm{~d} t \\
& =\frac{1-p^{N}}{1-p} \epsilon \int_{T_{1}}^{T_{0}} Q(t) \int_{t-\sigma-(N-1) \tau}^{T_{1}} Q(s) \mathrm{d} s \mathrm{~d} t \\
& \leqslant \frac{1-p^{N}}{1-p} \epsilon\left[\frac{3-4 p^{N}}{2\left(1-p^{N}\right)}(1-p) \int_{T_{1}}^{T_{0}} Q(s) \mathrm{d} s-\frac{1}{2}\left(\int_{T_{1}}^{T_{0}} Q(s) \mathrm{d} s\right)^{2}\right] \\
& =\frac{1-2 p^{N}}{1-p^{N}}(1-p) \epsilon
\end{aligned}
$$

Combining Cases 1 and 2, we have concluded that

$$
y\left(T_{0}\right) \leqslant \frac{1-2 p^{N}}{1-p^{N}}(1-p) \epsilon,
$$

which contradicts the fact that $y\left(T_{0}\right) \geqslant y(T)>\left(1-2 p^{N}\right)(1-p) \epsilon /\left(1-p^{N}\right)$. And therefore, the proof is complete.

In a similar way to Theorems 2.2 and 3.1 and applying Lemma 6.4.2 in [3], we can show the following asymptotic behaviour theorem.

Theorem 3.2. Assume that $|P(t)| \equiv p$ and there is a positive integer $N$ such that $4 p^{N} \leqslant 1$. If (1.3) and (1.12) hold, then every solution of equation (1.1) tends to zero as $t \rightarrow \infty$.

Acknowledgements. This work was supported by the National Natural Science Foundation of China and the Natural Sciences and Engineering Research Council of Canada.

\section{References}

1. G. GAO, On the $3 / 2$ asymptotic stability of one-dimensional functional differential equations with unbounded delay, Kexue Tongbao 33 (1993), 683-686 (in Chinese).

2. K. Gopalsamy, Stability and oscillations in delay differential equations of population dynamics (Kluwer Academic, Boston, 1992).

3. I. GYÖRI AND G. LADAS, Oscillation theory of delay differential equations with applications (Clarendon, Oxford, 1991).

4. V. B. KolmanovskiI, L. Torelli and R. Vermiglio, Stability of some test equations with delay, SIAM J. Math. Analysis 25 (1994), 948-961.

5. T. KRISZTin, On the stability properties for one-dimensional functional differential equations, Funkcial. Ekvac. 34 (1991), 241-256.

6. Y. KUANG, Delay differential equations with applications in population dynamics (Academic, Boston, 1993). 
7. M. R. S. Kulenovic, G. Ladas and A. Meimaridou, Stability of solutions of linear delay differential equations, Proc. Am. Math. Soc. 100 (1987), 433-441.

8. G. Ladas And Y. G. Sficas, Asymptotic behavior of oscillatory solutions, Hiroshima Math. J. 18 (1988), 351-359.

9. G. Ladas, Y. G. Sfichs and I. P. Stavroulakis, Asymptotic behavior of solutions of retarded differential equations, Proc. Am. Math. Soc. 88 (1983), 247-253.

10. J. W. H. So, J. S. YU AND M. P. CHEN, Asymptotic stability for scalar delay differential equations, Funkcial. Ekvac. 39 (1996), 1-17.

11. J. WU AND J. S. YU, Convergence in nonautonomous scalar neutral equations, Dyn. Syst. Applic. 4 (1995), 279-290

12. T. YoneyAma, On the $3 / 2$ stability theorem for one-dimensional delay-differential equations, J. Math. Analysis Applic. 125 (1987), 161-173.

13. T. Yoneyama, On the $3 / 2$ stability theorem for one-dimensional delay-differential equations with unbounded delay, J. Math. Analysis Applic. 165 (1992), 133-143.

14. J. A. Yorke, Asymptotic stability for one dimensional differential-delay equations, $J$. Diff. Eqns 7 (1970), 189-202.

15. J. S. YU, Asymptotic stability for nonautonomous scalar neutral differential equations, J. Math. Analysis Applic. 203 (1996), 850-860. 